\title{
Screening human donor corneas during organ culture for the presence of guttae
}

\author{
Vincent M Borderie, Visnja Sabolic, Olivier Touzeau, Sarah Scheer, \\ Santos Carvajal-Gonzalez, Laurent Laroche
}

\begin{abstract}
Aims-To detect the presence of guttae by means of light microscopy during organ culture and to evaluate the influence of the presence of guttae in the donor tissue on transplantation outcome.

Methods-Donor corneas were investigated for the presence of guttae by means of light microscopy at the end of organ culture. Recipient corneal buttons from patients with severe Fuchs' dystrophy and donor corneas with advanced guttae were first studied by light microscopy and subsequently by transmission electron microscopy. Lastly, 168 consecutive donor corneas were evaluated for the presence of guttae and issued for transplantation.

Results-Corneal specimens with Fuchs' dystrophy displayed numerous round highly reflecting guttae at the level of the corneal endothelium. Donor corneas with advanced guttae showed less numerous guttae. Among 168 organ cultured donor corneas issued for transplantation, low density guttae were found in $43(25.6 \%)$ corneas. The endothelial cell density and figure coefficient were significantly lower and organ culture time was significantly higher in the cornea guttata group than in the control group. The presence of grouped guttae significantly decreased the adjusted graft survival. The incidence of postoperative stage 3 cornea guttata was significantly higher when grouped guttae were found $(5 / 6)$ than when no guttae or scattered guttae were found (8/101).

Conclusion-Cornea guttata can be detected during organ culture by means of light microscopy. It is associated with a decrease in endothelial cell figure coefficient and cell density. The presence of grouped guttae is associated with poorer graft survival and more frequent stage 3 cornea guttata in the graft after transplantation.

(Br f Ophthalmol 2001;85:272-276)
\end{abstract}

Cornea guttata corresponds to a well defined corneal condition featuring the presence of focal thickenings of Descemet's membrane histologically named "guttae". ${ }^{1}$ Three clinical types of central corneal guttae are known: primary central corneal guttae, a common condition of the ageing cornea with no oedema throughout life, Fuchs' dystrophy in which corneal oedema occurs generally in the sixth decade, and secondary corneal guttae. Only a limited percentage of patients with cornea guttata correspond to patients with Fuchs' dystrophy. Cornea guttata can be easily diagnosed in vivo and ex vivo by means of specular microscopy as it gives dark areas where no endothelial cells are visible. ${ }^{2-4}$ Specular microscopy has been recommended to assess the donor corneal endothelium before transplantation for the presence of cornea guttata. ${ }^{5}$ During the organ culture procedure, the donor corneal endothelium is assessed by means of light microscopy after trypan blue staining and intercellular space dilatation. ${ }^{6}$ Light microscopy visualises not only the corneal endothelium surface but also the adjacent planes making corneal guttae more difficult to detect. Our objectives were to detect the presence of guttae by means of light microscopy during organ culture and to evaluate the influence of the presence of guttae in the donor tissue on transplantation outcome.

\section{Materials and methods}

STUDY DESIGN

Human donor corneas were investigated for the presence of cornea guttata by means of light microscopy during endothelial quality control at the end of organ culture. Five recipient corneal buttons from five patients with severe Fuchs' dystrophy (stage III Fuchs' dystrophy) ${ }^{3}$ were first studied by light microscopy after trypan blue staining and subsequently by routine transmission electron microscopy. Five donor corneas with advanced guttae (moderate stage II Fuchs' dystrophy) ${ }^{3}$ were then studied with the same methods. Lastly, 168 consecutive donor corneas were evaluated for the presence of guttae and studied by means of endothelial morphometric analysis. One hundred and sixty eight penetrating keratoplasties using these donor corneas carried out in 168 patients between April 1996 and January 1998 were prospectively studied.

LIGHT MICROSCOPY AND MORPHOMETRY The central endothelium was evaluated by induced dilatation of intercellular spaces and trypan blue $(0.3 \%)$ staining and was observed
Accepted for publication 18 September 2000 
Table 1 Cornea characteristics $(n=168)$

\begin{tabular}{|c|c|c|c|}
\hline & Mean $(S D)$ & Minimum & Maximum \\
\hline Donor age (years) & $68(14)$ & 32 & 94 \\
\hline Death to organ culture time (hours) & $39(10)$ & 11 & 48 \\
\hline Organ culture time (days) & $20.6(4.4)$ & 14 & 33 \\
\hline Endothelial density after organ culture (cells $/ \mathrm{mm}^{2}$ ) & $2126(272)$ & 1530 & 3060 \\
\hline $\begin{array}{l}\text { Coefficient of variation of endothelial cell area after } \\
\text { organ culture }(\%)\end{array}$ & $17.9(3.2)$ & 11.5 & 27.8 \\
\hline $\begin{array}{l}\text { Average figure coefficient of endothelial cells after organ } \\
\text { culture }(\%)\end{array}$ & $75(4)$ & 68 & 82 \\
\hline Deswelling time (days) & $2.3(2.5)$ & 1 & 4 \\
\hline
\end{tabular}

under a light microscope (Olympus BHS) coupled with a video camera interfaced to a frame buffer board operating in a Pentium computer. Images of the central endothelium were taken and endothelial morphometric analysis was performed using a Biocom image analysis system (Biocom, Les Ulis, France). One image of each cornea showing at least 100 cells with clear contours was analysed. For each digitised image, Visiolab 1000 software (Biocom) was used to analyse cells whose individual contours were obtained manually. The average figure coefficient, the average cell area, the coefficient of variation of cell area, and the endothelial density were calculated for each cornea. ${ }^{7}$

\section{CLINICAL STUDY}

Corneas were organ cultured at $31^{\circ} \mathrm{C}$ as previously described. ${ }^{7}$ The central endothelium was evaluated after organ culture. ${ }^{7}$ It was not evaluated before storage as we previously showed that endothelial evaluation before organ culture had no significant influence on graft postoperative outcome (that is, graft survival and postoperative endothelial cell density). ${ }^{7}$ Corneas were discarded for surgical use when they exhibited one or more of the following characteristics on light microscopic examination: an endothelial density of 1500 cells $/ \mathrm{mm}^{2}$ or less, severe cornea guttata, uncontinuous cellular mosaic, percentage of dead cells greater than $2 \%$ after organ culture, severe polymegethism or pleomorphism of the endothelial cell pattern. Donor age was not considered as a reason to discard corneas. After organ culture, corneas were incubated at room temperature with dextran containing medium. Cornea characteristics are shown in Table 1.

All transplants were performed at a single institution by three surgeons as previously described. ${ }^{78}$ The mean patient age was 59

Table 2 Patient characteristics $(n=168)$

\begin{tabular}{ll}
\hline Preoperative diagnosis: & \\
Fuchs' dystrophy and ICE syndrome & $18(10.7 \%)$ \\
Keratoconus & $34(20.2 \%)$ \\
Bullous keratopathy & $67(39.9 \%)$ \\
Other corneal dystrophies & $3(1.8 \%)$ \\
Corneal scar, corneal ulcer, interstitial keratitis & $19(11.3 \%)$ \\
Trauma & $4(2.4 \%)$ \\
Regraft & $23(13.7 \%)$ \\
Postoperative lens status: & $57(34.0 \%)$ \\
Phakic & $40(23.8 \%)$ \\
Posterior chamber IOL & $55(32.7 \%)$ \\
Anterior chamber IOL & $16(9.5 \%)$ \\
Aphakic & $129(76.8 \%)$ \\
Preoperative intraocular pressure: & $39(23.2 \%)$ \\
$\quad \leqslant 20$ mm Hg and no history of glaucoma & \\
>20 mm Hg and/or history of glaucoma & $120(71.4 \%)$ \\
Recipient rejection status: & $48(28.6 \%)$ \\
Low risk & \\
High risk & \\
\hline
\end{tabular}

years (SD 23, range 1-93 years). Patient characteristics are shown in Table 2. High risk recipients were defined as having a vascularised cornea (two or more quadrants of corneal vascularisation) or a history of irreversible corneal allograft rejection. Patients were hospitalised up to graft re-epithelialisation. They were then examined at 2 weeks, $1,3,6,9,12,18,24,30$, and 36 months after surgery. The average follow up time was 23.0 (SD 9.5) months. At 1 year postoperatively, 158 patients $(94.0 \%)$ were available for follow up, eight $(4.8 \%)$ were lost for follow up, and two $(1.2 \%)$ died. The criteria for graft failure were irreversible graft stromal oedema or corneal opacification. During the second year after transplantation contact wide field specular microscopy was performed in clear transplants of patients living in the country. The postoperative percentage endothelial cell loss was calculated as follows: ((endothelial cell density after preservation postoperative endothelial cell density)/ endothelial cell density after preservation) $\times$ 100 .

STATISTICAL ANALYSIS

The univariate effect of the presence of cornea guttata in the graft on continuous variables was studied using the Wilcoxon rank sum test. Its effect on categoric covariates was analysed with the $\chi^{2}$ test.

We studied unadjusted graft survival with the Kaplan-Meier method. The influence of the presence of cornea guttata in the graft on graft survival was studied using a multivariate Cox proportional hazards analysis including variables that were previously shown to influence graft survival (donor age, recipient age, recipient rejection status, and preoperative diagnosis). ${ }^{7}$ Survival curves according to the presence of cornea guttata in the graft were generated using a Cox model controlling for other covariates that were significant in the former model.

Visual acuity was studied by analysis of variance controlling for covariates that were previously shown to be significant (preoperative visual acuity, preoperative intraocular pressure, postoperative lens status, and postoperative astigmatism). ${ }^{8}$ Postoperative endothelial cell density and cell loss were studied by analysis of variance controlling for covariates that were previously shown to be significant (endothelial density after organ culture, coefficient of variation of endothelial cell area organ culture time, organ culture time, deswelling time, and preoperative diagnosis). ${ }^{7}$

\section{Results}

POSITIVE CONTROLS

Corneal specimens with Fuchs' dystrophy displayed numerous round highly reflecting guttae at the level of the corneal endothelium (Fig 1A). Only sparse endothelial cells could be seen between confluent guttae. Transmission electron microscopy (TEM) showed corneal injuries related to Fuchs' dystrophy (data not shown).

Donor corneas with advanced guttae showed the same light microscopic features as Fuchs' 
Table 3 Comparison of the cornea guttata group and the control group for preoperative variables

\begin{tabular}{llll}
\hline & $\begin{array}{l}\text { Control group } \\
(n=125)\end{array}$ & $\begin{array}{l}\text { Cornea guttata } \\
\text { group }(n=43)\end{array}$ & $\begin{array}{l}p \text { Value } \\
\text { (Wilcoxon rank sum test) }\end{array}$ \\
\hline Donor age (years) & $68(13)$ & $67(16)$ & $0.99(\mathrm{NS})$ \\
Death to organ culture time (hours) & $39(10)$ & $38(11)$ & $0.64(\mathrm{NS})$ \\
Organ culture time (days) & $19.9(4.0)$ & $22.5(5.1)$ & 0.003 \\
Endothelial density after organ culture (cells $\left./ \mathrm{mm}^{2}\right)$ & $2167(276)$ & $2006(224)$ & 0.0008 \\
Coefficient of variation of endothelial cell area after organ culture $(\%)$ & $18(3)$ & $18(2)$ & $0.50(\mathrm{NS})$ \\
Average figure coefficient of endothelial cells after organ culture $(\%)$ & $75(4)$ & $73(3)$ & 0.01
\end{tabular}

dystrophy corneas. However, guttae were less numerous and the endothelial mosaic could be seen between and over guttae. TEM showed excrescences of the Descemet's membrane.

CLINICAL STUDY

Among 168 organ cultured donor corneas issued for transplantation, low density guttae were found in $43(25.6 \%)$ corneas (cornea guttata group) whereas no guttae were found in the remaining $125(74.4 \%)$ corneas (control group) (Fig 1B). Cornea guttata could either be observed as sparse guttae between endothelial cells (Fig 1C), or grouped guttae forming clover figures, or grouped guttae within marked folds in Descemet's membrane (Fig 1D). The prevalence of guttae was $22 \%$ $(17 / 77)$ in corneas from male donors and $29 \%$ $(26 / 91)$ in corneas from female donors $(\mathrm{p}=$ 0.34 ). The cornea guttata group was divided into two subgroups according to the number of guttae per photograph. This resulted in three groups (Fig 1B-D) as follows: no guttae (group $1 ; \mathrm{n}=125$ ), one to three guttae per photograph (group $2 ; \mathrm{n}=35$ ), four guttae per photograph or more (group $3 ; n=8$ ). In group 2 only scattered guttae were observed, whereas in group 3 grouped guttae were observed.

The average endothelial cell density $(\mathrm{p}=$ $0.0008)$ and the average figure coefficient $(\mathrm{p}=$
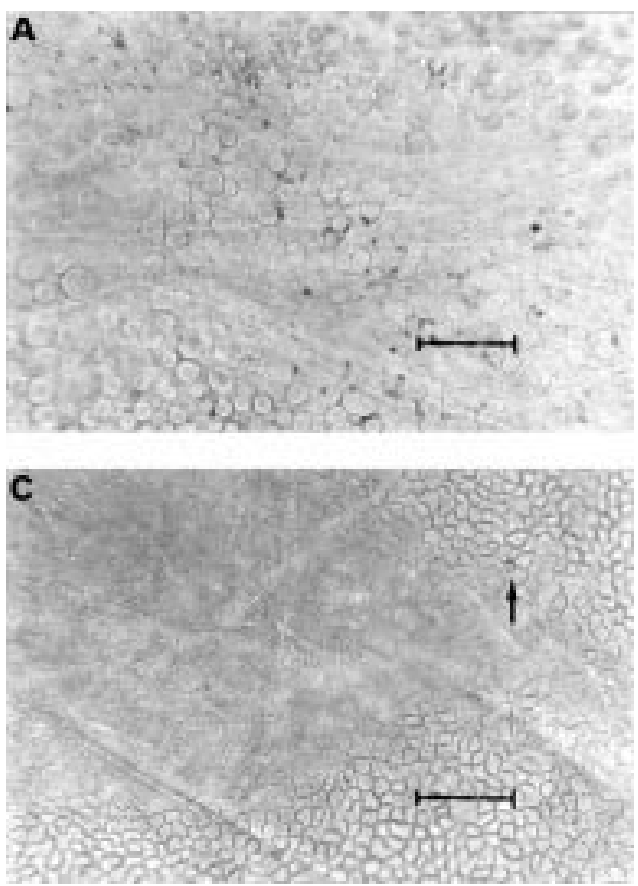

0.01) were significantly lower in the cornea guttata group than in the control group. The average organ culture time was significantly longer in the cornea guttata group than in the control group ( $p=0.003)$, whereas no significant differences for donor age, death to organ culture time, and the coefficient of variation of endothelial cell area were found between both groups (Table 3 ).

The overall graft survival was $88.1 \%$ at 12 months and $79.4 \%$ at 36 months (Fig 2). No significant differences in the adjusted graft survival were found between the cornea guttata group and the control group $(p=0.12)$ in the Cox model. However, when group 3 was compared with group $1+2$, the adjusted graft survival was significantly lower in the former group than in the latter group $(\mathrm{p}=0.007)$ (Fig 3). During the study period 28 grafts failed; 19 were immune failures and nine were nonimmune failures. The adjusted rejection-free graft survival was significantly lower in group 3 than in group $1+3(\mathrm{p}=0.0003)$.

No significant differences in postoperative visual acuity and postoperative graft thickness were found between the cornea guttata group and the control group in univariate analysis (Table 4) and by analysis of variance (data not shown). When group 3 was compared with
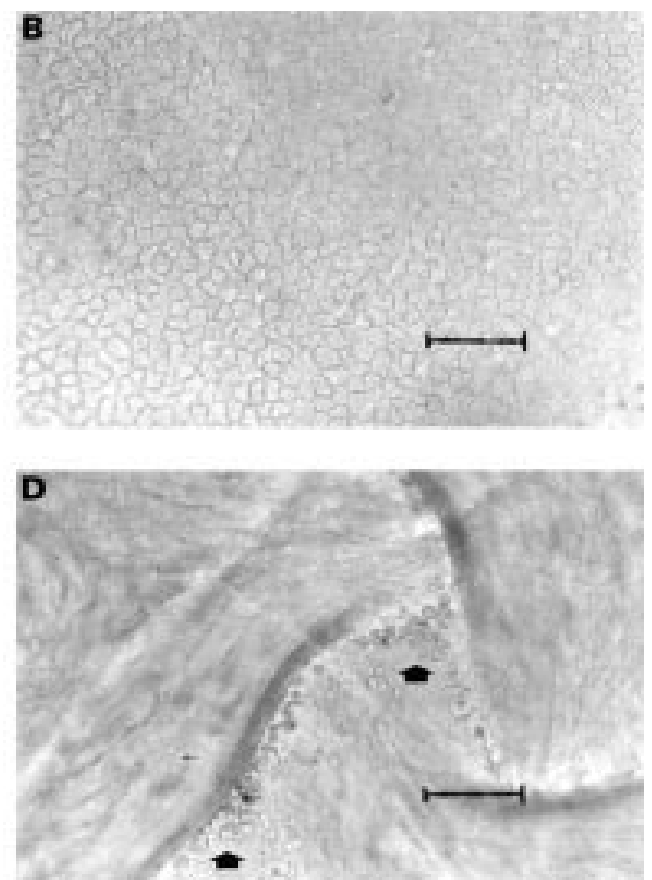

Figure 1 Light microscopy of the corneal endothelium and Descemet's membrane. (A) Recipient corneal button with stage III Fuchs' dystrophy showing numerous round highly reflecting guttae. (B) Normal donor cornea with no guttae (group 1). (C) Donor cornea with one isolated guttae (arrow) (group 2). (D) Donor cornea with grouped guttae (arrowheads) within a marked fold in Descemet's membrane, the remaining endothelial mosaic being normal (as in B) but not seen in the present micrograph because of the change in focus between the layers (group 3). Bars are $100 \mu \mathrm{m}$. 




Figure 2 Overall graft survival of 168 consecutive corneal grafts.

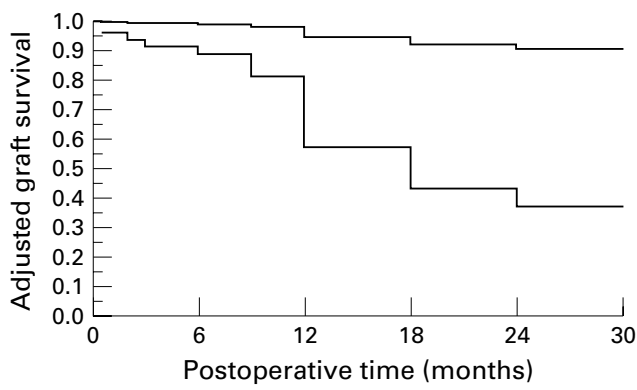

Figure 3 Influence of the presence of four guttae per photograph or more (grouped guttae) in the donor tissue (group 3; lower curve) on the adjusted graft survival. Donor tissue with no guttae or isolated guttae (group 1+2; upper curve) was associated with a significantly higher adjusted graft survival $(p=0.007)$.

group $1+2$, no significant differences in postoperative visual acuity at $12(\mathrm{p}=0.20), 18(\mathrm{p}=$ $0.47)$, and 24 months $(\mathrm{p}=0.64)$ and postoperative graft thickness at $12(\mathrm{p}=0.23)$, $18(\mathrm{p}=0.47)$, and 24 months $(\mathrm{p}=0.40)$ were found between both groups.

Among patients with a clear graft, specular microscopy was performed in 115 patients during the second postoperative year. No significant differences in postoperative endothelial cell density and postoperative endothelial cell loss were found between the cornea guttata group and the control group in univariate analysis (Table 4). However, analysis of variance controlling for other covariates showed the presence of guttae in the donor tissue $(p=0.01)$ to have a significant effect on the adjusted postoperative endothelial cell density and the adjusted postoperative endothelial cell loss (Table 5). When group 3 was compared with group $1+2$, no significant differences in postoperative endothelial cell density and postoperative endothelial cell loss were found between both groups in univariate analysis $(\mathrm{p}>$ $0.10)$ and by analysis of variance ( $p>0.80$ ). Stage 3 cornea guttata (guttae larger than an individual endothelial cell) ${ }^{2}$ was found by specular microscopy in 13 out of 115 grafts (11\%). The incidence of postoperative stage 3 cornea guttata was $83 \%(5 / 6)$ in group 3 and $7 \%(8 / 101)$ in group $1+2(p<0.00001)$.

\section{Discussion}

Cornea guttata can be detected during organ culture by means of light microscopy. Guttae consist of round highly reflecting structures and are observed at the level of the corneal endothelium. They can be differentiated from sloughing necrotic cells by gently moving the petri dish while observing the corneal endothelium. Sloughing necrotic cells move in a different pattern from the cornea (featuring oscillation movements) while guttae appear to be attached to the cornea. The high incidence $(5 / 6)$ of stage 3 cornea guttata (as diagnosed by specular microscopy) after transplantation in donor corneas with grouped guttae is a strong argument to be sure that the preoperative findings were actually guttae. Grouped guttae are often seen within marked folds in Descemet's membrane. This could be explained by impaired endothelial pump function in areas occupied by grouped guttae inducing fold formation.

Scattered guttae were found in $20.8 \%$ of 168 organ cultured corneas issued for transplantation. Grouped guttae were found in $4.8 \%$ of these 168 corneas. According to the mean donor age (68 (SD 14) years), this prevalence of cornea guttata in donor tissue is probably underestimated. In fact, the prevalence of cornea guttata was reported to be $70.4 \%$ in patients aged 40 years and more. ${ }^{9}$ Corneas with 1500 cells $/ \mathrm{mm}^{2}$ or less, severe cornea guttata, uncontinuous cellular mosaic, percentage of dead cells greater than $2 \%$, or severe polymegethism or pleomorphism of the endothelial cell pattern at the end of organ culture had been discarded. Then the overall frequency of cornea guttata in our donor corneas was probably higher than $26 \%$. No

Table 4 Comparison of the cornea guttata group and the control group for postoperative variables

\begin{tabular}{|c|c|c|c|}
\hline & $\begin{array}{l}\text { Control group } \\
(n=125)\end{array}$ & $\begin{array}{l}\text { Cornea guttata group } \\
(n=43)\end{array}$ & $\begin{array}{l}p \text { Value } \\
\text { (Wilcoxon rank sum test) }\end{array}$ \\
\hline Postoperative visual acuity at 12 months & $0.40(0.26)$ & $0.41(0.28)$ & 0.83 (NS) \\
\hline Postoperative visual acuity at 18 months & $0.46(0.29)$ & $0.45(0.26)$ & 0.88 (NS) \\
\hline Postoperative visual acuity at 24 months & $0.53(0.27)$ & $0.44(0.50)$ & 0.28 (NS) \\
\hline Postoperative graft thickness at 12 months $(\mu \mathrm{m})$ & $551(48)$ & $552(54)$ & 0.80 (NS) \\
\hline Postoperative graft thickness at 18 months $(\mu \mathrm{m})$ & $553(45)$ & $556(71)$ & 0.86 (NS) \\
\hline Postoperative graft thickness at 24 months ( $\mu \mathrm{m})$ & $560(49)$ & $557(61)$ & 0.92 (NS) \\
\hline Postoperative endothelial density during the second year $\left(\mathrm{cells} / \mathrm{mm}^{2}\right)$ & $1611(603)$ & $1522(526)$ & 0.42 (NS) \\
\hline Postoperative endothelial cell loss (\%) & $26(26)$ & $23(27)$ & 0.66 (NS) \\
\hline
\end{tabular}

Table 5 Comparison of the cornea guttata group and the control group for the adjusted postoperative endothelial density and cell loss

\begin{tabular}{llll}
\hline & & Control group \\
$(n=89)$ & $\begin{array}{l}\text { Cornea guttata } \\
\text { group }(n=26)\end{array}$ & $\begin{array}{l}p \text { Value } \\
(\text { ANOVA })\end{array}$ \\
\hline $\begin{array}{l}\left.\text { Adjusted average postoperative endothelial density during the second year (cells } / \mathrm{mm}^{2}\right) \\
\text { Adjusted average postoperative endothelial cell loss (\%) }\end{array}$ & $\begin{array}{l}1668 \\
24\end{array}$ & $\begin{array}{l}1091 \\
49\end{array}$ & 0.011 \\
\hline
\end{tabular}


influence of sex on the prevalence of guttae in donor corneas was found, which is in accordance with previous results. ${ }^{9}$

A recent study showed the presence of dark areas in the endothelial mosaic with no visible endothelial cells, by means of specular microscopy, in six human donor corneas, corresponding to subendothelial vacuoles in transmission electron microscopy. ${ }^{10}$ It may be that at least some scattered guttae observed on light microscopy could be such subendothelial vacuoles. However, vacuoles were not observed with transmission electron microscopy in our five human donor corneas with guttae on light microscopy. Subendothelial vacuoles are easily observed with the specular microscope as no light reflection occurs at the level of vacuoles as opposed to the normal endothelial cells. Conversely, they are difficult to visualise with the light microscope as the endothelial cells overlying vacuoles are seen and no defects (or dark areas) in the endothelial mosaic are evident. Necrotic endothelial cells featuring ruptured and folded cell membrane, vacuolated cytoplasm, and cell collapsus on stained flat mounted preparations of injured rabbit corneal endothelium were shown to correspond to type 4 events in specular microscopy. ${ }^{11}$ Type 4 events appear as large, coarsely granular, and bright on specular microscopy. It may be that at least some scattered guttae observed in light microscopy could be such necrotic cells as neighbouring cells often formed rosettes surrounding these scattered guttae (Fig 1C). However, it is notable that stage 2 guttae give isolated dark structures with adjacent cells forming rosettes in specular microscopy. ${ }^{2}$ Consequently, it is not possible to be sure that all donor corneas classified as corneas with scattered guttae were cornea guttata corneas or if at least some of them corresponded to corneas with dead sloughing endothelial cells.

In the present study, cornea guttata was associated with lower endothelial cell figure coefficient and cell density. Organ culture time was significantly longer in the cornea guttata group than in the control group. It is unlikely that guttae formation could occur during such a short period of time ( $\leqslant 1$ month). Conversely, guttae could be more easily observed after longer periods of organ culture. In fact, organ culture induces endothelial intercellular space dilatation and storage time dependent endothelial cell loss. ${ }^{12}$ Endothelial cells covering guttae (making guttae difficult to visualise by light microscopy) could detach during organ culture.

The presence of guttae had only a limited influence on transplantation outcome. Grafts with preoperative guttae had a lower endothelial cell density during the second year after transplantation. No significant influence on graft survival, postoperative visual acuity, and graft thickness was found. However, when we considered grafts with grouped guttae, the presence of grouped guttae had a significant influence on graft survival and on the incidence of postoperative stage 3 cornea guttata. It was associated with poorer graft survival and more frequent stage 3 cornea guttata in the graft after transplantation. Both findings are strong arguments not to use donor corneas with grouped guttae for penetrating keratoplasty. However, these corneas are still suitable for lamellar keratoplasty. Conversely, donor corneas with scattered guttae are suitable for penetrating as are corneas with no guttae if, otherwise, the endothelial cell density and vitality are satisfactory. It seems advisable not to transplant corneas with confluent guttae as they correspond to Fuchs' dystrophy corneas. ${ }^{3}$

In conclusion, cornea guttata can be detected during organ culture by means of light microscopy. It is associated with a decrease in endothelial cell figure coefficient and cell density. The presence of grouped guttae is associated with poorer graft survival and more frequent stage 3 cornea guttata in the graft after transplantation. Donor corneas featuring such injuries of Descemet's membrane should not be used for penetrating keratoplasty.

This work was supported by the Fondation Claude Bernard pour le développement des recherches biologiques et médicales dans les Hôpitaux de l'Assistance Publique à Paris.

1 Waring GO III, Mbekeani JN. Corneal dystrophies. In: Leibowitz HM, Waring GO III, editors. Corneal disorders. Clinical diagnosis and management. 2nd ed. Philadelphia: WB cal diagnosis and manag

2 Laing RA, Leibowitz HM, Oak SS, et al. Endothelial mosaic in Fuchs' dystrophy. A qualitative evaluation with the specular microscope. Arch Ophthalmol 1981;99:80-3.

3 Wilson SE, Bourne WM. Fuchs' dystrophy. Cornea 1988;7: $2-18$.

4 Borderie V, Baudrimont M, Bourcier T, et al. Cornea guttata et dystrophie de Fuchs. F Fr Ophtalmol 1999;22:987-1002.

5 Brooks AM, Grant G, Gillies WE. The preoperative assessBrooks AM, Grant G, Gillies WE. The preoperative assess-
ment of the corneal endothelium. Aust NZ F Ophthalmol ment of the corn

6 Pels E, Schuchard Y. Organ culture in the Netherlands. In: Brightbill FS, ed. Corneal surgery. Theory, technique and tissue. 2nd ed. St Louis: Mosby, 1993: 622-32.

7 Borderie VM, Scheer S, Touzeau O, et al. Donor corneal tissue selection before penetrating keratoplasty. $\mathrm{Br} \mathcal{F}$ Ophthalmol 1998;82:382-8.

8 Borderie VM, Touzeau O, Allouch C, et al. The results of successful penetrating keratoplasty using donor organcultured corneal tissue. Transplantation 1999;67:1433-8.

9 Lorenzetti DW, Uotila MH, Parikh N. Central cornea guttata. Incidence in the general population. Am $\mathcal{f}$ Ophthal-
grention mol 1967;64:1155-8.

10 Nartey IN, Cavanagh HD, Jester JV, et al. Characterization of specular "dark events" in human donor corneal endothelium by scanning and transmisssion electron microscopy. Cornea 1998;17:544-9.

11 Sherrard ES. Characterization of changes observed in the corneal endothelium with the specular microscope. Invest Ophthalmol Vis Sci 1978;17:322-6.

12 Borderie VM, Kantelip B, Delbosc B, et al. Morphology, histology and ultrastructure of human C31 organ-cultured corneas. Cornea 1995;14:300-10. 